\title{
Quantitative X-ray Photoelectron Spectroscopy Imaging for Small Feature Compositional Screening
}

\author{
D.J. Surman ${ }^{1}$, S.C. Page ${ }^{2}$ A.J. Roberts ${ }^{2}$ S.J. Coultas ${ }^{2}$ \\ ${ }^{1}$ Kratos Analytical Inc., 100 Red Schoolhouse Rd, Chestnut Ridge, New York 10977, USA \\ ${ }^{2}$ Kratos Analytical Ltd, Wharfside, Trafford Wharf Road, Manchester, M17 1GP. UK
}

X-ray photoelectron spectroscopy (XPS) is an established technique for surface characterization where information originates from the upper $10 \mathrm{~nm}$ of the sa mple surface. XPS has the further advantage of being a quantitative technique. Modern state-of-the-art spectrometers are capable of data acquisition from small, selected areas of th e specimen where the analysis area is defined by either using a focused $\mathrm{x}$-ray excitation source or collecting photoelectrons from a selected area defined using an ape rture/iris in the electrostatic lens column, som etimes termed a virtual probe. Using either of these approaches to define a small analysis area limits the smallest possible analysis area to $c a$. $10 \mu \mathrm{m}$ using ISO definition of the $80 \% / 20 \%$ decrease in the signal [1]. However, although approximately $90 \%$ of the si gnal will arise from within the selected area, Baer et.al.[2] demonstrated that information is collec ted from up to three tim es the defined area which could compromise interpretation of data.

As an alternative to selected area XPS outlined above this paper presents the im plementation and application of quantitative XPS imaging which has become possible with the recent im plementation of pulse counting 2D detectors for XPS i maging. There are a number of advantages to using quantitative imaging over the more conventional scanned spectrum. When acquiring spectra it must be assumed that the are a sampled is homogeneous which in reality may or may not be the case. By acquiring quantitative images eith er elemental or chemical distribution of the species at the surface can be observed with a spatial resolution in the order of $3 \mu \mathrm{m}$. Furthermore, combining fast imaging with stage movements allows the acquisition of high resolution XPS im ages over areas of several $\mathrm{mm}^{2}$.

The spherical mirror analyzer (SMA) incorporated in to a standard laboratory spectrometer allows fast parallel images to be acquired whereby the spatial resolution of the photoelectrons at the surface is preserved as they are transm itted through the analyzer. I mportantly the SMA works in fi xed analyzer transmission mode, such that th e energy resolution rem ains constant as a function of binding (or kinetic) energy. This becom es very important when requiring to quantify im ages and is analogous to the operation of the hemispherical analyzer used in normal spectroscopy mode.

The familiar photoelectron spectrum com prises a series of peaks corres ponding to the elem ents at the surface of the sample. These peaks sit on a background of inelas tically scattered photoelectrons which generally increases with increasing binding energy. The relative atom ic concentration of the elements at the surface is calcu lated using the area under the peak after subtracting the background, and correcting for a relative sen sitivity factor and instrument transmission. To generate a quantitative image, the same steps must be undert aken whereby each im age acquired for a specific element must have a background im age subtracted and be corrected for the re lative sensitive factor and instrument transm ission. In generating an im age that bette $\mathrm{r}$ describes the background for a specific photoelectron peak, im ages are acquired e ither side of the phot oemission peak itself and a linearly interpolated image calculated which is then subtracted from the peak image. This is shown 
in both im aging mode and the equivalent spectr oscopic mode in figure 1. A test sam ple was produced where bulk PTFE polymer was covered $\mathrm{w}$ ith a mask and the surface was $\mathrm{m}$ odified by exposure to a propanal plasm a creating a patte rned sample with hydrocarbon islands on a fluorocarbon substrate. Using th e imaging technique outlined above quantitative images were generated for hydrocarbon, fluorocarbon, oxygen and fluor ine, shown in figure 2. Quantification of the elemental composition was found to be dependent on acquisition tim e of the im ages but for a total acquisition time for all images of $405 \mathrm{~s}$ the quantification was in good agreement with spectra acquired from a $55 \mu \mathrm{m}$ diameter analysis area. The advantag e of the im aging approach to surface composition determination is apparent from the images where it can be seen that the coverage of the area exposed to the propanal is not homogeneous. Post acquisition the software [3] is used to define small areas within the plasma treated area to generate the relative atomic concentration.

Quantitative imaging has been demonstrated to be useful way to charac terize the surface chemistry of samples. This app roach allows the qu antification from areas sm aller than possible with traditional selected area spectroscopy whilst also be identifying areas of inhomogeneity which might otherwise be overlooked in spectroscopy mode.

References:

[1] ISO 18115:2001

[2] D.R. Baer, M.H. Engelhard, Surf. Int. Anal., 29, 766-772 (2000).

[3] CasaXPS processing software, CasaXPS Ltd, UK www.casaxps.com

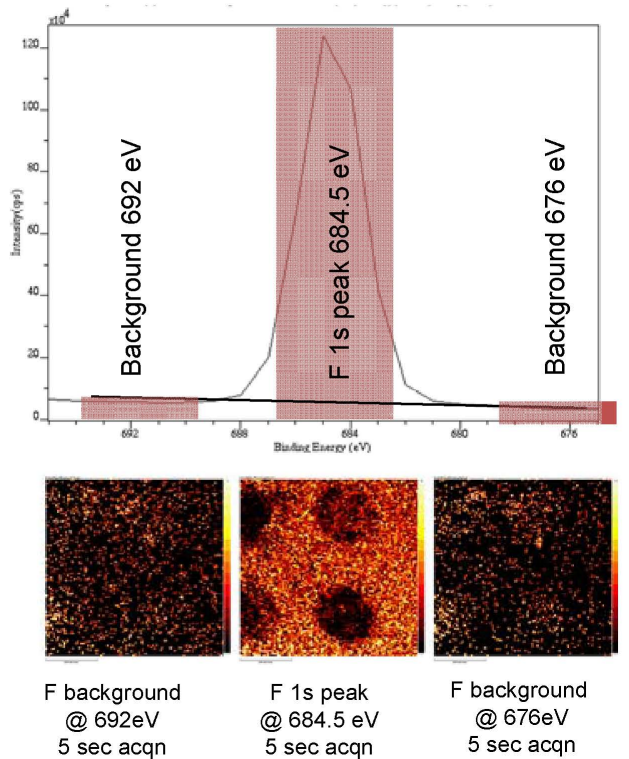

Fig. 1. Example of peak and background images and equivalent for spectra for patterned sample.
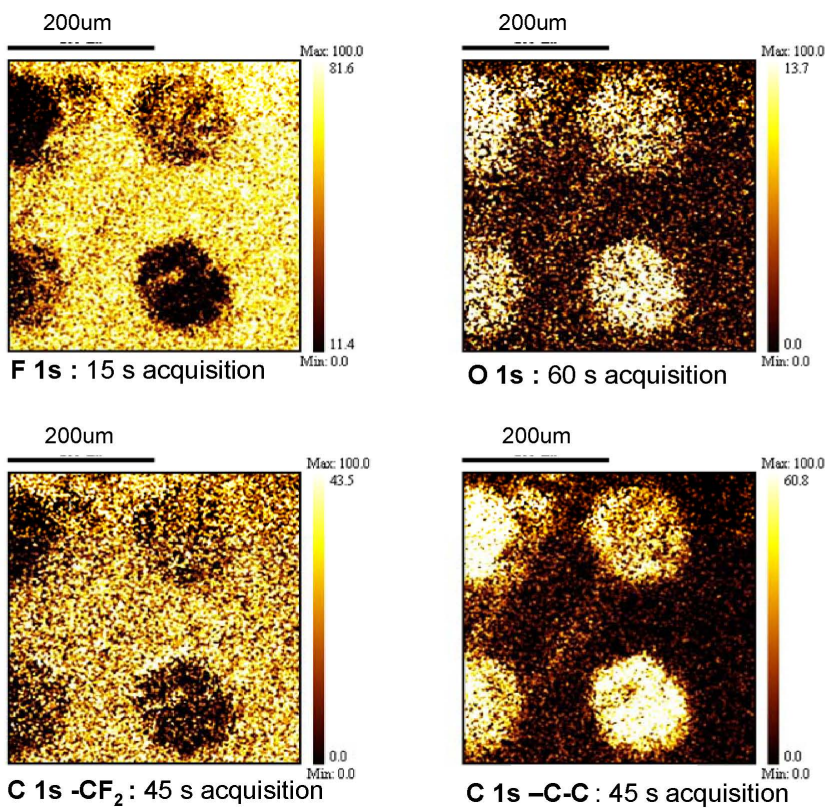

Fig. 2. Quantitative elemental and chemical state images from propanal plasma polymer patterned sample on PTFE substrate. 